\title{
Spin-orbit coupling affecting the evolution of transverse spin
}

\author{
Jörg S. Eismann $\odot,{ }^{*}$ Peter Banzer $\odot$, and Martin Neugebauer $\odot$ \\ Max Planck Institute for the Science of Light, Staudtstrasse 2, D-91058 Erlangen, Germany \\ and Institute of Optics, Information, and Photonics, University Erlangen-Nuremberg, Staudtstrasse 7/B2, D-91058 Erlangen, Germany
}

(Received 1 July 2019; published 3 December 2019)

\begin{abstract}
We investigate the evolution of transverse spin in tightly focused circularly polarized beams of light, where spin-orbit coupling causes a local rotation of the polarization ellipses upon propagation through the focal volume. The effect can be explained as a relative Gouy-phase shift between the circularly polarized transverse field and the longitudinal field carrying orbital angular momentum. The corresponding rotation of the spatial distribution of the electric transverse spin density is observed experimentally by utilizing a recently developed reconstruction scheme, which relies on transverse-spin-dependent directional scattering of a nanoprobe.
\end{abstract}

DOI: 10.1103/PhysRevResearch.1.033143

\section{INTRODUCTION}

The investigation of spin-orbit interactions of light has become an integral field in modern optics, with a huge variety of related effects being relevant for many applications [1]. Spin-orbit coupling plays an important role in the design of spin-dependent metasurfaces [2-4], liquid crystal mode converters [5,6], and directional waveguide and plasmon couplers [7-9], etc. Furthermore, it is of relevance in the field of super-resolution microscopy in the context of proper depletion beams $[10,11]$ and in optical manipulation experiments $[12,13]$.

The spin-orbit coupling also occurs naturally when a circularly polarized beam is focused [1]. The arising orbital angular momentum can thereby be described by a geometric Berryphase effect [14], where the longitudinal component of the field accumulates a phase of $2 \pi$ for one trip around the optical axis $[1,15]$. The corresponding focal field distributions and their properties regarding spin and orbital angular momentum have been investigated in various works over the past decade [1,16-18].

In this article, we report on an effect which links the three-dimensional distribution of the spin density (SD) to the orbital part of the spin-orbit-coupled beam. Again, the effect occurs when an initially circularly polarized collimated beam of light is tightly focused. For simplicity, we consider a Gaussian beam profile, although the results can be generalized for higher order modes. Because of the aforementioned spinorbit coupling, the focused beam carries not only spin but also orbital angular momentum, which arises in the form of a phase vortex of the longitudinal field component $[13,15]$. The superposition of the longitudinal field and the circularly

\footnotetext{
*joerg.eismann@mpl.mpg.de; http://www.mpl.mpg.de/

Published by the American Physical Society under the terms of the Creative Commons Attribution 4.0 International license. Further distribution of this work must maintain attribution to the author(s) and the published article's title, journal citation, and DOI.
}

polarized transverse field results in a tilted polarization ellipse offside the optical axis. Consequently, the corresponding SD features transverse components with respect to the propagation direction of the beam (optical axis). The actual local orientation of the spin depends on the relative phase between the longitudinal and transverse fields, which changes upon propagation [19]. As we will show later on, this causes the spatial distribution of the transverse components of the SD to rotate while traversing the focal region.

In the following, we start by describing the aforementioned effect as a Gouy-phase-dependent interaction of longitudinal and transverse fields [20,21]. For this, we elaborate on a simplified theoretical model in the framework of an extended paraxial approximation considering also longitudinal field components. To demonstrate the rotation of the transverse SD experimentally, we use a recently developed scheme for measuring transversely spinning fields in tightly focused light beams [22,23]. Finally, we compare our results with numerical calculations and discuss the possible implications of the effect on future works.

\section{THEORETICAL MODEL}

We begin with a simplified paraxial description of a timeharmonic circularly polarized Gaussian beam, propagating along the $z$ axis. Utilizing the complex beam parameter $q(z)=$ $z-\imath z_{R}$, where $z_{R}$ is the Rayleigh range of the beam, the field components are described by [24]

$$
\left(\begin{array}{c}
E_{x} \\
E_{y} \\
E_{z}
\end{array}\right)=\left(\begin{array}{c}
1 \\
\pm l \\
0
\end{array}\right) \frac{u_{0}}{q(z)} e^{\frac{l k \rho^{2}}{2 q(z)}+l k z}=\sigma^{ \pm} u(\mathbf{r})
$$

with radius $\rho=\sqrt{x^{2}+y^{2}}, u_{0}$ a complex amplitude, and the wave number $k$. The sign of the polarization vector $\sigma^{ \pm}=$ $(1, \pm \imath, 0)$ indicates right- or left-handed circular polarization. However, the field distribution as described by Eq. (1) does not fulfill Gauss's law in vacuum, $\nabla \mathbf{E}=0$, which requires an additional longitudinal field component. Within the paraxial approximation, the missing component can be calculated 
using [25]

$$
E_{z}^{ \pm}=\frac{l}{k}\left(\frac{\partial E_{x}}{\partial x}+\frac{\partial E_{y}}{\partial y}\right)=-\frac{u(\mathbf{r})}{q(z)}(x \pm \imath y) .
$$

For a more intuitive description of the distribution of $E_{z}$, we rewrite Eq. (2) using the azimuth $\phi=\arg (x+\imath y)$ and the Gouy phase $\eta(z)=\tan ^{-1}\left(z / z_{R}\right)$ :

$$
E_{z}^{ \pm}=-\rho \frac{l u(\mathbf{r})}{\sqrt{z^{2}+z_{R}^{2}}} e^{ \pm \imath \phi-\imath \eta(z)} .
$$

As we can see, $E_{z}$ is represented by a first-order LaguerreGaussian mode with radial mode index 0 and azimuthal mode index \pm 1 (the sign depends on the handedness of the incoming circularly polarized beam). Thus, the longitudinal field exhibits the helical phase-front typically associated with the occurrence of orbital angular momentum [26]. Furthermore, in comparison to the transverse field (zeroth-order Laguerre-Gaussian mode), $E_{z}$ exhibits an additional Gouyphase factor $[19,21,26]$. Therefore, the relative phase between longitudinal and transverse fields changes upon propagation, which consequently affects the three-dimensional polarization state.

Here, we take a closer look at the evolution of the SD of the electric field $\mathbf{s}_{E}$, which describes the local orientation and sense of the spinning axis of the three-dimensional polarization ellipse $[1,27,28]$. For our paraxial model, $\mathbf{s}_{E}$ is described by the following equation:

$$
\mathbf{s}_{E}^{ \pm}=\frac{\epsilon_{0} \operatorname{Im}\left[\mathbf{E}^{*} \times \mathbf{E}\right]}{4 \omega}=\left(\begin{array}{c}
-\frac{\rho \sin [\phi \mp \eta(z)]}{\sqrt{z^{2}+z_{R}^{2}}} \\
\frac{\rho \cos [\phi \mp \eta(z)]}{\sqrt{z^{2}+z_{R}^{2}}} \\
\pm 1
\end{array}\right) \frac{\epsilon_{0}|u(\mathbf{r})|^{2}}{2 \omega} .
$$

The longitudinal component of the SD has exactly the same Gaussian distribution as the electric field intensity distributions $\left|E_{x}\right|^{2}=\left|E_{y}\right|^{2} \propto s_{E}^{z}$ whereby the sign indicates right- and left-handed circular polarizations. Most importantly it is shape invariant upon propagation. In contrast, the shapes of the transverse SD components depend on the Gouy phase and, as a consequence, also on $z$. In particular, the distributions of $s_{E}^{x}$ and $s_{E}^{y}$ rotate upon propagation along the $z$ axis. From $z=-\infty$ to $z=\infty$, the SD vector undergoes one half-twist around the $z$ axis. The rotation direction depends on the handedness of the incoming circular polarization.

For a more intuitive understanding, the effect is visualized in Fig. 1, where we consider a right-handed circularly polarized beam propagating top down, with the local spin density marked as blue vectors and the corresponding orientation and spinning direction of the electric field indicated by black arrows. In the far field of the upper half-space $(z<0)$, the spin points toward the geometrical focus. A projection onto the $x-y$ plane reveals that the transverse SD, $\mathbf{s}_{E}^{\perp}=s_{E}^{x} \mathbf{e}_{x}+s_{E}^{y} \mathbf{e}_{y}$, is pointing toward the optical axis (see top right inset). Upon propagation, the relative phase between the transverse and longitudinal field components changes, which results in a rotation of the transverse SD. In the focal plane, $\mathbf{s}_{E}^{\perp}$ exhibits a purely azimuthal distribution (see central inset). Below the focal plane $(z>0)$, the rotation continues, finally reaching a

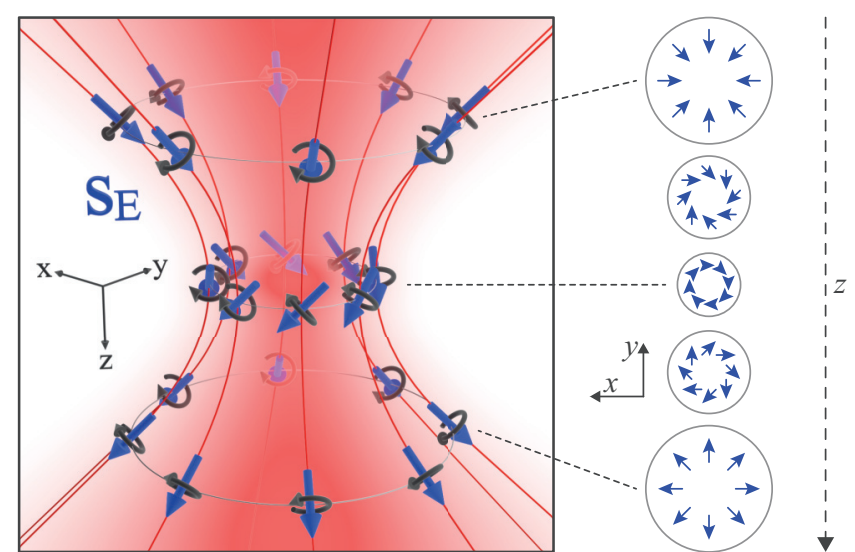

FIG. 1. Illustration of the electric spin density $\mathbf{s}_{E}$ distribution of a tightly focused right-handed circularly polarized beam. The beam (red) propagates top down, with the blue vectors indicating the local orientation of $\mathbf{s}_{E}$ and the black vectors corresponding to the polarization ellipse of the electric field $\mathbf{E}$. The sketches on the right side represent the transverse spin density $\mathbf{s}_{E}^{\perp}$ for different planes of observation.

radial distribution pointing away from the optical axis in the far field (lowest inset).

In order to confirm the half-twist of the spin density, we elaborate on this phenomenon with an experimental demonstration and numerical calculations.

\section{EXPERIMENTAL OBSERVATION}

First, we investigate the rotation of the SD experimentally. Since the strength of the transverse spin depends on the lateral confinement [27], we investigate a circularly polarized beam (wavelength $\lambda=532 \mathrm{~nm}$ ) tightly focused by a high numerical aperture microscope objective (NA $=0.9$, pupil filling factor $\approx 0.8$ ). The beam impinges onto a dipole-like gold nanosphere (radius $\approx 40 \mathrm{~nm}$ ) sitting on a glass substrate, which is scanned through a focal volume of $\approx 3 \times 3 \times 3 \mu \mathrm{m}^{3}$, where we use a step size of $30 \mathrm{~nm}$ in lateral directions ( $x$ and $y$ ) and steps of $200 \mathrm{~nm}$ along the propagation direction $(z)$. For each position, the light scattered into the glass half space is collected with an index-matched immersion-type objective. The directionality of the scattered light into the substrate allows for determining the transverse SD of the excitation field at the particle position [22,23]. This is due to the fact that the directional scattering is a direct consequence of the spinning electric dipole induced in the particle by the locally transverse components of the SD [27]. Finally, we assemble the scanning results to three-dimensional representations of the transverse SD components $s_{E}^{x}$ and $s_{E}^{y}$. The measurement results for right- and left-handed circularly polarized incoming beams are depicted in Figs. 2(a) and 2(b) and Figs. 2(f) and $2(\mathrm{~g})$, respectively. As predicted by the paraxial model and Eq. (4), the distributions of the transverse SD components rotate upon propagation, with the rotation direction depending on the handedness of the incoming circular polarization. This verifies the coupling of the transverse SD distribution and the longitudinal orbital angular momentum. For a 

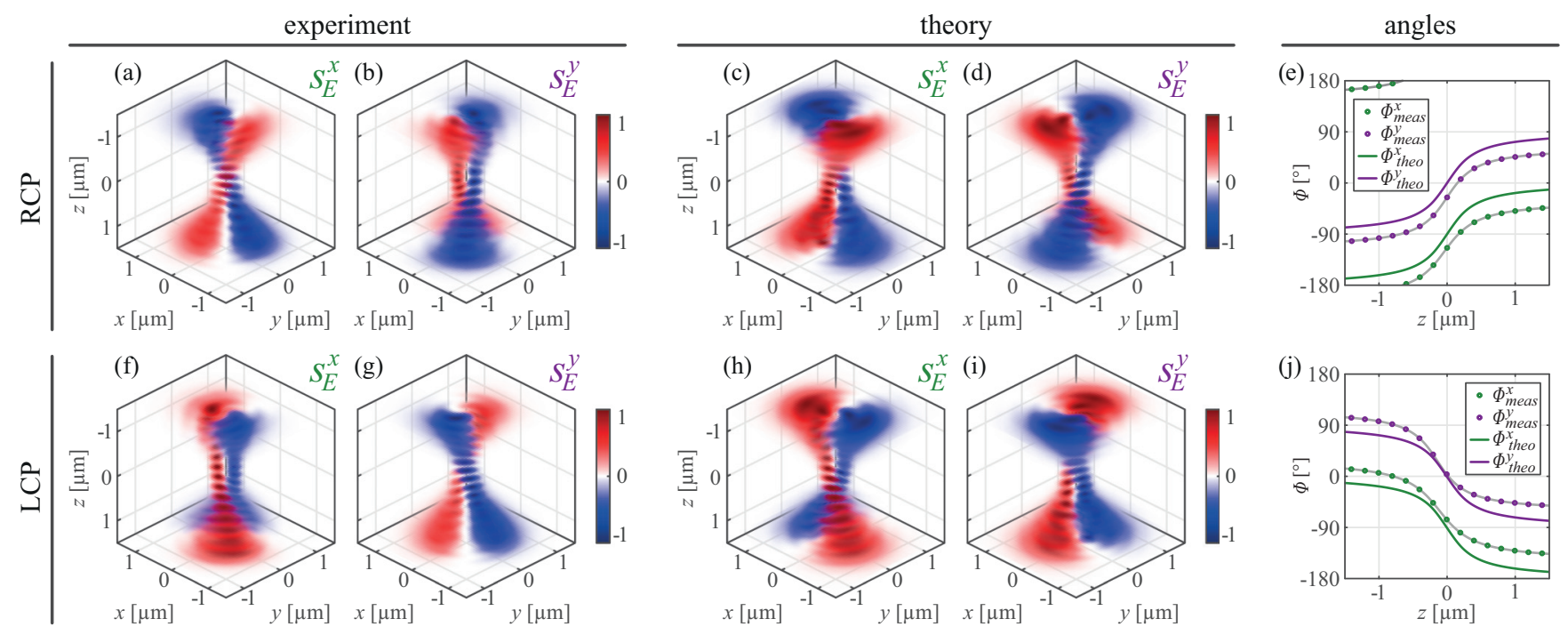

FIG. 2. Experimental and theoretical results. [(a)-(d)] The experimentally measured and theoretically calculated transverse SD components $s_{E}^{x}$ and $s_{E}^{y}$ in the focal volume of a tightly focused right-handed circularly polarized (RCP) beam. For each plane of observation along the $z$ axis, the transverse SD is normalized to its maximum amplitude for better visibility. (e) Experimental (green and purple circles) and theoretical (green and purple lines) rotation angles of the transverse SD distributions calculated from the distributions in panels (a)-(d). The Gouy-phase factor is fitted to the experimental data (gray lines). [(f)-(j)] Similar to panels (a)-(e), but for a left-handed circularly polarized (LCP) beam.

quantitative comparison, we calculate the corresponding transverse SD distributions using vectorial diffraction theory $[29,30]$, where we use the same parameters as in the experiment and consider the beam to be in free space (effects of the glass substrate on the field distributions are not taken into account). The theoretical results are shown in Figs. 2(c) and 2(d) and Figs. 2(h) and 2(i). All four theoretical distributions are in very good agreement with their corresponding experimental counterparts. Also the rotation of the transverse spin densities predicted by the simplified paraxial model is confirmed by the vectorial diffraction theory. As a next step, we determine the rotation angles of the distributions of $s_{E}^{x}$ and $s_{E}^{y}$. For this purpose, we calculate the centroids of the positive and negative parts of the respective SD component for each $x-y$ plane of observation along the $z$ axis and define the angle between the connection line of both centroids and the $x$ axis as rotation angle $\phi(z)$. The theoretical (solid lines) and experimental (circles) rotation angles are plotted in Figs. 2(e) and $2(\mathrm{j})$, where the green and purple colors correspond to $s_{E}^{x}$ and $s_{E}^{y}$, respectively. For the right-handed circularly polarized beam, the experimentally measured rotation angles exhibit a negative angular offset with respect to the theoretical curves, while for the left-handed circularly polarized beam the offset is positive. This spin-dependent offset is caused by the interference of the incoming beam and the light reflected by the glass substrate, which is not considered in the theoretical treatment. Still, the rotation angles follow a modified inverse tangent function, $\phi(z)= \pm \tan ^{-1}\left[\left(z+z_{o}\right) / z_{R}\right]+\phi_{o}$, with $z_{o}$ being the offset along the $z$ axis and $\phi_{o}$ being the angular offset. The fits, which overlap very well with the experimental data, are plotted as gray lines. This verifies that even in the tight focusing regime, we can use the relative Gouy-phase factor between longitudinal and transverse fields derived from the paraxial model as a qualitative explanation for the half-twist of the SD.

\section{DISCUSSION}

In conclusion, we observed the rotation of the transverse SD and its spatial distribution upon propagation in tightly focused circularly polarized Gaussian beams. The effect can be explained by a difference in mode orders and, therefore, a relative Gouy-phase between the transverse field components and the longitudinal field, which carries orbital angular momentum due to spin-orbit coupling. In this regard, the measurement of the rotation of the transverse spin density can also be interpreted as an experimental demonstration of the nonseparability of three-dimensional fields [31,32], and the generation of orbital angular momentum by tight focusing $[1,16,17]$.

The evolution of three-dimensional polarization states upon propagation is relevant in polarization-based metrology and microscopy approaches [33], where the local polarization state entails information on the position of a scatterer relative to an excitation field [34-36]. By utilizing a more complex input field distribution, it is possible to tailor the rotation of the SD along a given axis in space [19], which might facilitate the practical implementation of position-sensing techniques and spin-based directional coupling experiments, where the directionality strongly depends on the local orientation of the spin $[8,9]$. Furthermore, the notion of a position dependent orientation of the SD in tightly focused circularly polarized beams can be relevant for optical manipulation experiments, with the local spin exerting a torque or a lateral force on nanoparticles [37,38].

Whenever circularly polarized light is highly confined, transverse components of the spin density occur, which locally tilt the spin away from the propagation axis. Our work demonstrates that the direction of this tilt changes upon propagation, even in a fundamental circularly polarized Gaussian beam not requiring any artificially structured input beams. 
Importantly, the presented concept can also be adopted for circularly polarized higher order Laguerre Gaussian modes $[6,39,40]$. There again, the mode orders of the longitudinal and transverse field components differ by \pm 1 , resulting in a half-twist of the spatial distribution of the transverse SD similar to the one discussed here for the fundamental Gaussian mode. This renders the presented effect highly relevant for any spin-dependent nano-optics experiment.

\section{ACKNOWLEDGMENTS}

We gratefully acknowledge discussions with Sergey Nechayev and Gerd Leuchs. This project has received funding from the European Commission Horizon 2020 research and innovation programme under the Future and Emerging Technologies Open Grant Agreement Super-Pixels No. 829116.
[1] K. Y. Bliokh, F. J. Rodríguez-Fortuño, F. Nori, and A. V. Zayats, Nat. Photon. 9, 796 (2015).

[2] N. Shitrit, I. Yulevich, E. Maguid, D. Ozeri, E. Veksler, V. Kleiner, and E. Hasman, Science 340, 724 (2013).

[3] E. Karimi, S. A. Schulz, I. De Leon, H. Qassim, J. Upham, and R. W. Boyd, Light. Sci. Appl. 3, e167 (2014).

[4] X. Ling, X. Zhou, X. Yi, W. Shu, Y. Liu, S. Chen, H. Luo, S. Wen, and D. Fan, Light. Sci. Appl. 4, e290 (2015).

[5] L. Marrucci, C. Manzo, and D. Paparo, Phys. Rev. Lett. 96, 163905 (2006).

[6] F. Cardano, E. Karimi, L. Marrucci, C. de Lisio, and E. Santamato, Opt. Express 21, 8815 (2013).

[7] J. Lin, J. P. B. Mueller, Q. Wang, G. Yuan, N. Antoniou, X.-C. Yuan, and F. Capasso, Science 340, 331 (2013).

[8] F. J. Rodríguez-Fortuño, G. Marino, P. Ginzburg, D. O'Connor, A. Martínez, G. A. Wurtz, and A. V. Zayats, Science 340, 328 (2013).

[9] M. Neugebauer, T. Bauer, P. Banzer, and G. Leuchs, Nano Lett. 14, 2546 (2014)

[10] E. Rittweger, K. Y. Han, S. E. Irvine, C. Eggeling, and S. W. Hell, Nat. Photon. 3, 144 (2009).

[11] X. Hao, C. Kuang, T. Wang, and X. Liu, J. Opt. 12, 115707 (2010).

[12] H. Adachi, S. Akahoshi, and K. Miyakawa, Phys. Rev. A 75, 063409 (2007).

[13] Y. Zhao, J. S. Edgar, G. D. M. Jeffries, D. McGloin, and D. T. Chiu, Phys. Rev. Lett. 99, 073901 (2007).

[14] R. Bhandari, Phys. Rep. 281, 1 (1997).

[15] Z. Bomzon and M. Gu, Opt. Lett. 32, 3017 (2007).

[16] T. A. Nieminen, A. B. Stilgoe, N. R. Heckenberg, and H. Rubinsztein-Dunlop, J. Opt. A: Pure Appl. Opt. 10, 115005 (2008).

[17] K. Y. Bliokh and F. Nori, Phys. Rep. 592, 1 (2015).

[18] D. Sugic and M. R. Dennis, J. Opt. Soc. Am. A 35, 1987 (2018).

[19] X. Pang and W. Miao, Opt. Lett. 43, 4831 (2018).

[20] L. G. Gouy, C. R. Acad. Sci. (Paris) 110, 1251 (1890).

[21] X. Pang and T. D. Visser, Opt. Express 21, 8331 (2013).
[22] M. Neugebauer, T. Bauer, A. Aiello, and P. Banzer, Phys. Rev. Lett. 114, 063901 (2015).

[23] M. Neugebauer, J. S. Eismann, T. Bauer, and P. Banzer, Phys, Rev. X 8, 021042 (2018).

[24] Q. Zhan, Adv. Opt. Photon. 1, 1 (2009).

[25] W. L. Erikson and S. Singh, Phys. Rev. E 49, 5778 (1994).

[26] D. L. Andrews and M. Babiker, The Angular Momentum of Light, 1st ed. (Cambridge University Press, Cambridge, UK, 2013).

[27] A. Aiello, P. Banzer, M. Neugebauer, and G. Leuchs, Nat. Photon. 9, 789 (2015).

[28] T. Bauer, M. Neugebauer, G. Leuchs, and P. Banzer, Phys. Rev. Lett. 117, 013601 (2016).

[29] B. Richards and E. Wolf, Proc. R. Soc. London A 253, 358 (1959).

[30] L. Novotny and B. Hecht, Principles of Nano-optics, 2nd ed. (Cambridge University Press, Cambridge, UK, 2006).

[31] A. Holleczek, A. Aiello, C. Gabriel, C. Marquardt, and G. Leuchs, Opt. Express 19, 9714 (2011).

[32] X.-F. Qian and J. H. Eberly, Opt. Lett. 36, 4110 (2011).

[33] A. Goto, K. Otomo, and T. Nemoto, Frontiers Phys. 7, 56 (2019).

[34] S. Berg-Johansen, T. Falk, B. Stiller, P. Banzer, M. Ornigotti, E. Giacobino, G. Leuchs, A. Aiello, and C. Marquardt, Optica 2, 864 (2015).

[35] Z. Xi, L. Wei, A. J. L. Adam, H. P. Urbach, and L. Du, Phys. Rev. Lett. 117, 113903 (2016).

[36] A. Bag, M. Neugebauer, P. Woźniak, G. Leuchs, and P. Banzer, Phys. Rev. Lett. 121, 193902 (2018).

[37] A. Canaguier-Durand, A. Cuche, C. Genet, and T. W. Ebbesen, Phys. Rev. A 88, 033831 (2013).

[38] F. J. Rodríguez-Fortuño, N. Engheta, A. Martínez, and A. V. Zayats, Nat. Commun. 6, 8799 (2015).

[39] A. M. Beckley, T. G. Brown, and M. A. Alonso, Opt. Express 18, 10777 (2010).

[40] M. Neugebauer, S. Grosche, S. Rothau, G. Leuchs, and P. Banzer, Opt. Lett. 41, 3499 (2016). 